\title{
Adaptabilidade e estabilidade via regressão não paramétrica em genótipos de café
}

\author{
Moysés Nascimento(1), Adésio Ferreira(2), Romário Gava Ferrão(3), Ana Carolina Mota Campana(1), \\ Leonardo Lopes Bhering(4), Cosme Damião Cruz $^{(5)}$, Maria Amélia Gava Ferrão ${ }^{(3)}$ \\ e Aymbiré Francisco Almeida da Fonseca(6)
}

\begin{abstract}
(1)Universidade Federal de Viçosa (UFV), Departamento de Estatística, Avenida P.H. Rolfs, s/no, CEP 36571-000 Viçosa, MG. E-mail: moysesnascim@ufv.br, carolcampana@vicosa.ufv.br (2)Universidade Federal do Espírito Santo, Alto Universitário, s/non, CEP 29500-000 Alegre, ES. E-mail: adesioferreira@gmail.com ${ }^{(3)}$ Instituto Capixaba de Pesquisa, Assistência Técnica e Extensão Rural (Incaper), Rua Afonso Sarlo, no 160, CEP 29052-010 Vitória, ES. E-mail: romario@incaper.es.gov.br, mferrao@incaper.es.gov.br (4)Embrapa Agroenergia, Parque Estação Biológica, Embrapa Sede, s/no, CEP 70770-901 Brasília, DF. E-mail: leonardo.bhering@embrapa.br ${ }^{(5)}$ UFV, Departamento de Biologia Geral. E-mail: cdcruz@ufv.br ${ }^{(6)}$ Embrapa Café, Parque Estação Biológica, Embrapa Sede, s/no CEP 70770-901 Brasília, DF. E-mail: aymbire@incaper.es.gov.br
\end{abstract}

Resumo - O objetivo deste trabalho foi avaliar uma metodologia de análise de adaptabilidade e estabilidade fenotípica de genótipos de café baseada em regressão não paramétrica. A técnica utilizada difere das demais, pois reduz a influência na estimação do parâmetro de adaptabilidade de algum ponto extremo, ocasionado pela presença de genótipos com respostas demasiadamente diferenciadas a determinado ambiente. Foram utilizados dados provenientes de um experimento sobre produtividade média de grãos de 40 genótipos de café (Coffea canephora), com delineamento em blocos ao acaso, com seis repetições. Os genótipos foram avaliados em cinco anos (1996, 1998, 1999, 2000 e 2001), em dois locais (Sooretama e Marilândia, ES) no total de dez ambientes. A metodologia proposta demonstrou ser adequada e eficiente, pois extingue os efeitos impróprios induzidos pela presença de pontos extremos e evita a recomendação incorreta de genótipos quanto à adaptabilidade.

Termos para indexação: Coffea canephora, análise estatística, interação genótipo x ambiente, melhoramento genético, pontos extremos.

\section{Adaptability and stability based on nonparametric regression in coffee genotypes}

\begin{abstract}
The objective of this work was to evaluate a methodology of phenotypic adaptability and stability analyses of coffee genotypes based on nonparametric regression. The technique used differs from other techniques because it reduces the influence of extreme points resulting from the presence of genotypes whose answers to a certain environment are too different on the estimation of the adaptability parameter. Data from an experiment studying the average yield of 40 coffee (Coffea canephora) genotypes in a randomized block design with six replicates were used to evaluate the method. The genotypes were evaluated along five years $(1996,1998,1999,2000$ and 2001) in two locations (Sooretama and Marilândia, ES, Brazil), in a total of ten environments. The methodology proposed proved adequate and efficient, since it eliminates the disproportionate effects induced by the presence of extreme points and avoids misleading recommendations of genotypes in terms of adaptability.
\end{abstract}

Index terms: Coffea canephora, statistical analysis, genotype x environment interaction, crop breeding, outliers.

\section{Introdução}

Nos programas de melhoramento, na fase de seleção e, principalmente, na fase de recomendação, o conhecimento do componente de interação genótipo x ambiente é de grande importância. Porém, não fornece informações pormenorizadas sobre o comportamento de cada cultivar diante das variações ambientais (Cruz et al., 2004). Assim, as análises de adaptabilidade e de estabilidade tornam-se necessárias para a identificação e recomendação de materiais superiores em diferentes ambientes.

As metodologias de análise de adaptabilidade e estabilidade são diferentes quanto aos conceitos adotados e a certos princípios estatísticos empregados 
(Cruz et al., 2004). Como exemplo, tem-se as metodologias baseadas em regressão linear simples (Eberhart \& Russell, 1966), regressão linear múltipla (Cruz et al., 1989), análises não paramétricas (Lin \& Binns, 1988; Carneiro, 1998), centroides múltiplos e centroide ampliado (Nascimento et al., 2009a, 2009b).

Apesar da grande quantidade de metodologias, nenhuma trata da existência de pontos extremos, isto é, de situações nas quais genótipos têm resposta variada a determinado ambiente. Assim, trabalhos que utilizaram a metodologia de Eberhart \& Russell (1966), como os de Vasconcelos et al. (2008), Miranda et al. (2009), Pereira et al. (2009), e não levaram em consideração a presença de pontos extremos e, se presentes, esses pontos desconsiderados podem ter influenciado as análises. O mesmo vale para os trabalhos de Albrecht et al. (2007), Porto et al. (2007) e Matsuo et al. (2008), nos quais foi utilizada a metodologia de Lin \& Binns (1988).

As metodologias baseadas em análise de regressão, seja ela simples ou bissegmentada, por exemplo, sofrem a ação de pontos extremos em razão do processo de estimação. Isso pode proporcionar estimativas inadequadas, que não refletem a verdadeira relação existente entre a variação ambiental e a resposta genotípica, e superestimar ou subestimar o parâmetro de adaptabilidade. As metodologias de Lin \& Binns (1988) e Carneiro (1998), de avaliação da performance genotípica com base em medidas não paramétricas, também podem ocasionar resultados impróprios na presença de pontos discrepantes, pois o valor dos parâmetros pode ser inflacionado e provocar classificação incorreta do genótipo quanto a adaptabilidade e estabilidade. Quando se consideram as metodologias dos centroides múltiplos e centroide ampliado (Nascimento et al., 2009a, 2009b), baseadas em componentes principais, a presença de pontos discrepantes influencia diretamente a configuração gráfica obtida por meio dos escores dos genótipos. Assim, genótipos afetados por pontos discrepantes têm um comportamento diferenciado dos demais genótipos no gráfico da análise, o que ocasiona a sua incorreta classificação.

Quando se deparam com esse problema, os pesquisadores geralmente não os consideram ou optam pela exclusão de ambiente(s), o que pode levar a inadequadas recomendações com a primeira conduta e a perdas de informações, tempo, recursos financeiros e trabalho com a segunda.

O objetivo deste trabalho foi avaliar uma metodologia de análise de adaptabilidade e estabilidade fenotípica de genótipos de café baseada em regressão não paramétrica.

\section{Material e Métodos}

Para a avaliação da metodologia, foram utilizados dados do programa de melhoramento de café do Instituto Capixaba de Pesquisa, Assistência Técnica e Extensão Rural (Incaper), ES. Os dados são provenientes da avaliação da produtividade média de grãos $\left(\mathrm{kg} \mathrm{ha}^{-1}\right)$ de 40 genótipos de Coffea canephora avaliados durante cinco anos (1996, 1998, 1999, 2000 e 2001) em dois locais (Sooretama e Marilândia, ES), no total de dez ambientes. Os experimentos foram implantados e conduzidos sem irrigação, no delineamento experimental de blocos ao acaso, com seis repetições. A avaliação da adaptabilidade e estabilidade dos genótipos também foi realizada pelas metodologias de Eberhart \& Russell (1966) e de Lin \& Binns (1988).

Para utilização do método proposto em um experimento com genótipos (g), ambientes (a) e repetições (r), definiu-se o modelo estatístico $Y_{\mathrm{ij}}=\beta_{0 \mathrm{i}}+\beta_{\mathrm{li}} \mathrm{I}_{\mathrm{j}}+\psi_{\mathrm{ij}}$, em que: $Y_{\mathrm{ij}}$ é a média de genótipo i no ambiente $\mathrm{j} ; \beta_{0 \mathrm{i}}$ é o coeficiente linear referente ao i-ésimo genótipo (intercepto); $\beta_{\mathrm{li}}$ é o coeficiente de regressão, o qual mede a resposta do i-ésimo genótipo à variação do ambiente; $\mathrm{I}_{\mathrm{j}}$ é o índice ambiental codificado $I_{j}=\left(\sum_{j} Y_{j} / g\right)-\left(\sum_{i} \sum_{j} Y_{i j} / g a\right)$; $\psi_{\mathrm{ij}}$ são os erros aleatórios provenientes de uma população com mediana zero.

As estimativas de $\mathrm{I}_{\mathrm{j}}$ indicam a qualidade do ambiente, em que valores negativos identificam ambientes desfavoráveis e valores positivos, ambientes favoráveis.

Na metodologia proposta, o conceito de adaptabilidade refere-se à capacidade de os genótipos responderem ao estímulo do ambiente: genótipos com adaptabilidade ampla ou geral, $\beta_{\mathrm{li}}=1$; genótipos com adaptabilidade específica a ambientes favoráveis, $\beta_{\mathrm{li}}>1$; genótipos com adaptabilidade específica a ambientes desfavoráveis, $\beta_{\mathrm{li}}<1$. O conceito de estabilidade refere-se à capacidade de os genótipos mostrarem um comportamento 
previsível em função do estímulo do ambiente. Foram utilizados dois parâmetros para medir a estabilidade, $\mathrm{R}_{\mathrm{T}_{\mathrm{i}}}^{2}$ e $\mathrm{R}_{\mathrm{T}_{\mathrm{i}}}^{* 2}$. A partir desses parâmetros, os genótipos foram classificados em: genótipos com estabilidade ou previsibilidade alta, $\mathrm{R}_{\mathrm{T}_{\mathrm{i}}}^{2}$ ou $\mathrm{R}_{\mathrm{T}_{\mathrm{i}}}^{* 2}$ maior que $70 \%$; genótipos com estabilidade ou previsibilidade baixa, $\mathrm{R}_{\mathrm{T}_{\mathrm{i}}}^{2}$ ou $\mathrm{R}_{\mathrm{T}_{\mathrm{i}}}^{* 2}$ menor que $70 \%$.

A estimação do coeficiente angular $\beta_{\mathrm{li}}$, parâmetro de adaptabilidade via regressão não paramétrica, foi realizada da seguinte forma: calcularam-se todos os valores amostrais $\mathrm{A}=[\mathrm{a}(\mathrm{a}-1)] / 2$ de $\mathrm{S}_{\mathrm{ikl}}=\left(\mathrm{Y}_{\mathrm{il}}-\mathrm{Y}_{\mathrm{ik}}\right) /$ $\left(\mathrm{I}_{\mathrm{l}}-\mathrm{I}_{\mathrm{k}}\right)$ para todo $\mathrm{i}=1, \ldots$, g e $1 \leq \mathrm{k}<1 \leq \mathrm{a}$, em que $\mathrm{Y}_{\mathrm{il}}$ é a média referente ao i-ésimo genótipo no l-ésimo ambiente; $\mathrm{I}_{1}$ é o valor do índice ambiental referente ao 1-ésimo ambiente. Assim o estimador de $\beta_{\mathrm{li}}$ é dado por $\hat{\beta}=$ mediana $\left\{\mathrm{S}_{\mathrm{ik}}, 1 \leq \mathrm{k}<1 \leq \mathrm{a}\right\}$. Então, se $\mathrm{A}$ é ímpar, $\mathrm{A}=2 \mathrm{k}+1$, tem-se $\hat{\beta}=\mathrm{S}^{\mathrm{k}+1}$. Se A é par, $\mathrm{A}=2 \mathrm{k}$, tem-se $\hat{\beta}_{1 \mathrm{i}}=\left(\mathrm{S}^{\mathrm{k}}+\mathrm{S}^{\mathrm{k}+1}\right) / 2$.

A avaliação da hipótese $\mathrm{H}_{0 \mathrm{i}}: \beta_{\mathrm{li}}=1$ foi realizada por meio do teste t, cuja estatística é dada por $\mathrm{t}=\left(\hat{\beta}_{1 \mathrm{i}}-1\right) / \sqrt{\hat{\mathrm{V}}\left(\hat{\beta}_{1 \mathrm{i}}\right)}$, a qual está associada ao nível de significância $\alpha$ e ao número de graus de liberdade do resíduo, obtidos na análise conjunta. A variância de $\beta_{\mathrm{li}}$ foi dada por $\sigma_{\mathrm{e}}^{2}=\mathrm{QMR} / \mathrm{r}$, válido para todo i, em que: $\sigma_{\mathrm{e}}^{2}=\mathrm{QMR} / \mathrm{r}$ e $\mathrm{QMR}=\hat{\sigma}^{2}$ é o quadrado médio do resíduo da análise conjunta e $r$ é numero de repetições que deram origem às médias submetidas à análise de adaptabilidade e estabilidade.

Theil (1950) sugeriu duas formas de estimação do intercepto $\beta_{0 \mathrm{i}}$ : estimar pela mediana de todos $\hat{\beta}_{0 \mathrm{i}}=\mathrm{y}_{\mathrm{ij}}-\hat{\beta}_{1 \mathrm{i}} \mathrm{I}_{\mathrm{j}}$ ou por $\hat{\beta}=\operatorname{mediana}\left(\mathrm{y}_{\mathrm{ij}}\right)-\hat{\beta}_{\mathrm{li}}$ mediana $\left(\mathrm{I}_{\mathrm{j}}\right)$ (Sprent \& Smeeton, 2000). Neste trabalho, foi utilizada a primeira sugestão.

$\mathrm{Na}$ metodologia proposta, a estimação do parâmetro de estabilidade foi feita a partir de um dos dois parâmetros: com base no coeficiente de determinação, como em mínimos quadrados (equação 1), ou estimada pelo quociente (equação 2). $\mathrm{R}_{\mathrm{T}_{\mathrm{i}}}^{2}=\left(\mathrm{SQ}_{\text {Total }}-\mathrm{SQ}_{\text {Erros }}\right) / \mathrm{SQ}_{\text {Total }}$

Em razão da falta da propriedade de ortogonalidade no modelo, a soma de quadrados total não pode ser decomposta em soma do quadrado da regressão mais soma do quadrado do resíduo, o que pode causar estimativas negativas com o primeiro parâmetro. $\mathrm{R}_{\mathrm{T}_{\mathrm{i}}}^{* 2}=\operatorname{Var}\left(\hat{\mathrm{y}}_{\mathrm{ij}}\right) / \operatorname{Var}\left(\mathrm{y}_{\mathrm{ij}}\right)$

Entretanto, existia a questão de como saber se há um ponto extremo que esteja superestimando ou subestimando o parâmetro de adaptabilidade. Para resolvê-la, definiu-se como medida da influência de um ponto a variação, em módulo, entre os estimadores do coeficiente de inclinação estimados pelos métodos de mínimos quadrados $\left(\hat{\beta}_{\mathrm{li}}^{*}\right)$ e pelo método de regressão não paramétrica $\left(\hat{\beta}_{\mathrm{li}}\right)$ para cada genótipo, isto é: $\Delta \hat{\beta}_{1 \mathrm{i}}=\left|\hat{\beta}_{1 \mathrm{i}}^{*}-\hat{\beta}_{\mathrm{1i}}\right|$. Assim, quanto maior essa variação, maior o indicativo da influência do ponto discrepante em questão. Neste trabalho, foi considerado o valor de 0,05 para declarar a existência de um ponto extremo. Para casos em que $\Delta \hat{\beta}_{\mathrm{li}}$ não ultrapassou o valor definido, realizou-se a análise pelos métodos tradicionais. As análises estatísticas foram realizadas pelo programa Genes (Cruz, 2006).

\section{Resultados e Discussão}

$\mathrm{Na}$ análise de variância conjunta para rendimento de grãos, observou-se diferença significativa $(p<0,01)$ na interação genótipos $\mathrm{x}$ ambientes pelo teste $\mathrm{F}$ (Tabela 1), o que indica haver comportamento diferenciado dos genótipos nos ambientes avaliados. Esse fato dificulta a recomendação de cultivares, pois não se pode, nessas circunstâncias, fazer uma recomendação uniforme para todos os locais sem prejuízo considerável para a produção. Dessa forma, torna-se justificável e necessária a utilização de técnicas de estimação de adaptabilidade e estabilidade.

Os genótipos ES 306, ES 307, ES 310, ES 312, ES 314, ES 315, ES 319, ES 320, ES 321, ES 322, ES 323, ES 327 , ES 330, ES 331, ES 335, ES 337, ES 339, ES 340, ES 36, ES 01, VCPe VSM apresentaram valores de $\Delta \hat{\beta}_{\mathrm{li}}$ iguais ou superiores a 0,05 , tendo sido, portanto, considerados influenciados por pontos extremos (Tabela 2).

A comparação das estimativas dos parâmetros de adaptabilidade e estabilidade pela metodologia de Eberhart \& Russell (1966) com a de regressão não paramétrica (Tabela 2) demonstrou que os genótipos ES 315, ES 319, ES 323, ES 327, ES 340, ES 36, ES 01, VCP e VSM são discrepantes quanto às classificações, e

Tabela 1. Análise de variância conjunta de dez experimentos, com 40 genótipos avaliados em seis blocos por ensaio, quanto ao caráter produção média de grãos de café.

\begin{tabular}{lrc}
\hline Fonte de variação & GL & QMR \\
\hline Blocos/ambientes & 30 & $409.611,09$ \\
Genótipo & 39 & $11.068 .510,89^{* *}$ \\
Ambientes & 9 & $170.086 .397,96^{* *}$ \\
Genótipo x ambiente & 351 & $2.351 .135,76^{* *}$ \\
Resíduo & 1.170 & $361.768,50$ \\
\hline CV (\%) & & 22,34 \\
\hline
\end{tabular}

**Significativo a $1 \%$ pelo teste $\mathrm{F}$. 
revelou a ocorrência de recomendação incorreta quando não são levados em consideração os pontos extremos.

Os genótipos ES 315, ES 319, ES 323, ES 36 e VCP foram classificados como de adaptabilidade geral quando avaliados pelo método Eberhart \& Russell (1966). Entretanto, quando foram analisados com base na metodologia proposta no presente trabalho, esses genótipos foram classificados como de adaptabilidade específica a ambientes desfavoráveis. Os genótipos ES 340 e VSM, classificados como de adaptabilidade geral pelo método de Eberhart \& Russell (1966), foram classificados como de adaptabilidade específica a ambientes favoráveis pelo método proposto. O genótipo ES 327 foi classificado como de adaptabilidade específica a ambientes desfavoráveis por meiodeEberhart\&Russell(1966), ecomodeadaptabilidade geral pela metodologia não paramétrica proposta. Com base na metodologia de Eberhart \& Russell (1966), o genótipo ES 01 foi classificado como de adaptabilidade específica a ambientes favoráveis enquanto, na metodologia não paramétrica, foi classificado como de adaptabilidade geral. A influência exercida pelos pontos discrepantes é menor no

Tabela 2. Estimativas dos parâmetros de adaptabilidade e estabilidade em genótipos de café pelos métodos de Eberhart \& Russell (1966) e de regressão não paramétrica ${ }^{(1)}$.

\begin{tabular}{|c|c|c|c|c|c|c|c|c|}
\hline Genótipo & $\Delta \hat{\beta}_{1 \mathrm{i}}=\left|\hat{\beta}_{1 \mathrm{i}}^{*}-\hat{\beta}_{1 \mathrm{i}}\right|$ & $\hat{\beta}_{0 \mathrm{i}}^{*}$ & $\hat{\beta}_{0 \mathrm{i}}$ & $\hat{\beta}_{1_{i}}^{*}$ & $\hat{\beta}_{1 \mathrm{i}}$ & $\mathrm{R}_{\mathrm{T}_{\mathrm{i}}}^{2}$ & $\mathrm{R}_{\mathrm{ER}_{\mathrm{i}}}^{2}$ & $\sigma_{\mathrm{d}_{\mathrm{i}}}^{2}$ \\
\hline ES 306 & 0,10 & $2.027,94$ & $1.897,79$ & $0,71 * *$ & $0,61 *$ & 53,06 & 54,28 & $423.693,39 * *$ \\
\hline ES 307 & 0,13 & $2.889,75$ & $2.815,86$ & $1,57 * *$ & $1,69^{*}$ & 74,17 & 74,65 & $906.689,18^{* *}$ \\
\hline ES 308 & 0,02 & $2.795,90$ & $2.681,10$ & $1,03^{\mathrm{ns}}$ & $1,05^{\mathrm{ns}}$ & 45,48 & 45,50 & $1.430 .097,74^{* *}$ \\
\hline ES 309 & 0,04 & $2.878,66$ & $2.679,16$ & $0,42 * *$ & $0,38^{*}$ & 13,89 & 14,02 & $1.200 .496,60 * *$ \\
\hline ES 310 & 0,10 & $3.059,26$ & $3.394,12$ & $0,93^{\mathrm{ns}}$ & $1,03^{\mathrm{ns}}$ & 57,24 & 57,93 & $656.344,82 * *$ \\
\hline ES 311 & 0,02 & $2.786,42$ & $2.868,33$ & $1,02^{\mathrm{ns}}$ & $1,00^{\mathrm{ns}}$ & 91,69 & 91,71 & $22.249,11^{\mathrm{ns}}$ \\
\hline ES 312 & 0,08 & $2.534,10$ & $2.370,76$ & $0,72 * *$ & $0,64 *$ & 65,23 & 66,04 & $230.707,08 * *$ \\
\hline ES 313 & 0,01 & $2.817,98$ & $2.901,44$ & $1,24^{*}$ & $1,25^{*}$ & 85,46 & 85,47 & $221.830,90^{* *}$ \\
\hline ES 314 & 0,16 & $3.002,80$ & $2.772,18$ & $1,12^{\text {ns }}$ & $0,96^{\mathrm{ns}}$ & 70,64 & 72,06 & $487.162,90 * *$ \\
\hline ES 315 & 0,07 & $2.135,27$ & $2.166,58$ & $0,90^{\text {ns }}$ & $0,83 * *$ & 81,77 & 82,21 & $118.190,16^{*}$ \\
\hline ES 316 & 0,04 & $2.736,44$ & $2.969,80$ & $0,79 *$ & $0,83 * *$ & 61,57 & 61,73 & $367.737,62 *$ \\
\hline ES 317 & 0,01 & $2.383,15$ & $2.361,80$ & $0,88^{\mathrm{ns}}$ & $0,87^{\mathrm{ns}}$ & 89,19 & 89,21 & $22.514,40^{\mathrm{ns}}$ \\
\hline ES 318 & 0,00 & $1.352,13$ & $1.361,91$ & $0,27 * *$ & $0,26^{* *}$ & 54,84 & 54,85 & $-20.690,38^{\mathrm{ns}}$ \\
\hline ES 319 & 0,33 & $2.681,05$ & $2.527,28$ & $1,11^{\mathrm{ns}}$ & $0,78^{* *}$ & 58,61 & 64,29 & $730.644,89 * *$ \\
\hline ES 320 & 0,11 & $2.910,58$ & $2.773,91$ & $1,42 * *$ & $1,31 *$ & 83,52 & 84,04 & $369.843,81 * *$ \\
\hline ES 321 & 0,13 & $2.816,59$ & $2.665,46$ & $0,98^{\text {ns }}$ & $0,85^{\mathrm{ns}}$ & 69,42 & 70,75 & $385.927,12 * *$ \\
\hline ES 322 & 0,05 & $2.364,67$ & $2.371,30$ & $1,04^{\mathrm{ns}}$ & $0,99^{\text {ns }}$ & 81,40 & 81,59 & $200.812,36^{* *}$ \\
\hline ES 323 & 0,15 & $2.168,12$ & $2.011,04$ & $0,83^{\text {ns }}$ & $0,68^{*}$ & 46,37 & 47,92 & $801.271,86^{* *}$ \\
\hline ES 324 & 0,02 & $2.074,83$ & $2.084,95$ & $0,73 * *$ & $0,75^{*}$ & 79,47 & 79,56 & $72.614,32^{\mathrm{ns}}$ \\
\hline ES 325 & 0,03 & $2.744,88$ & $2.658,01$ & $1,07^{\mathrm{ns}}$ & $1,10^{\mathrm{ns}}$ & 88,42 & 88,48 & $88.049,28^{*}$ \\
\hline ES 326 & 0,01 & $2.791,69$ & $2.884,12$ & $1,37 * *$ & $1,39 *$ & 67,31 & 67,32 & $1.003 .647,63 * *$ \\
\hline ES 327 & 0,15 & $3.235,27$ & $3.168,93$ & $0,76^{*}$ & $0,92^{\mathrm{ns}}$ & 39,13 & 40,79 & $919.664,87 * *$ \\
\hline ES 328 & 0,00 & $3.592,72$ & $3.552,83$ & $1,64 * *$ & $1,64^{*}$ & 90,99 & 90,99 & $228.192,38 * *$ \\
\hline ES 329 & 0,04 & $4.087,16$ & $3.924,81$ & $1,39 * *$ & $1,35^{*}$ & 77,00 & 77,06 & $593.661,16^{* *}$ \\
\hline ES 330 & 0,06 & $2.621,23$ & $2.287,61$ & $1,03^{\mathrm{ns}}$ & $0,96^{\mathrm{ns}}$ & 72,72 & 73,01 & $376.901,26 * *$ \\
\hline ES 331 & 0,11 & $2.628,48$ & $2.527,57$ & $0,68^{*}$ & $0,57^{*}$ & 60,97 & 62,71 & $239.845,49 * *$ \\
\hline ES 332 & 0,03 & $2.623,07$ & $2.514,33$ & $1,00^{\mathrm{ns}}$ & $0,97^{\mathrm{ns}}$ & 77,09 & 77,15 & $261.626,27 * *$ \\
\hline ES 333 & 0,02 & $2.073,81$ & $2.019,24$ & $0,88^{\mathrm{ns}}$ & $0,90^{\mathrm{ns}}$ & 82,29 & 82,33 & $106.534,20^{*}$ \\
\hline ES 334 & 0,03 & $2.726,63$ & $2.599,59$ & $1,17^{*}$ & $1,20 *$ & 78,76 & 78,80 & $351.358,81 * *$ \\
\hline ES 335 & 0,10 & $2.684,65$ & $2.743,13$ & $0,86^{\mathrm{ns}}$ & $0,96^{\mathrm{ns}}$ & 77,82 & 78,83 & $146.339,16^{* *}$ \\
\hline ES 336 & 0,02 & $3.591,51$ & $3.429,97$ & $0,89^{\text {ns }}$ & $0,91^{\mathrm{ns}}$ & 60,91 & 60,94 & $515.133,71^{* *}$ \\
\hline ES 337 & 0,05 & $3.507,93$ & $3.288,23$ & $1,18^{\mathrm{ns}}$ & $1,13^{\mathrm{ns}}$ & 50,54 & 50,62 & $1.532 .735,71 * *$ \\
\hline ES 338 & 0,01 & $2.338,82$ & $2.420,22$ & $0,74 * *$ & $0,74 * *$ & 82,17 & 82,17 & $51.043,81^{\mathrm{ns}}$ \\
\hline ES 339 & 0,10 & $3.183,27$ & $3.318,22$ & $1,36^{* *}$ & $1,46^{*}$ & 81,69 & 82,15 & $389.962,44^{* *}$ \\
\hline ES 340 & 0,05 & $2.985,46$ & $3.324,68$ & $1,15^{\mathrm{ns}}$ & $1,20 * *$ & 42,00 & 42,10 & $2.074 .096,75^{* *}$ \\
\hline ES 36 & 0,05 & $1.903,26$ & $1.716,63$ & $0,86^{\text {ns }}$ & $0,81 * *$ & 69,95 & 70,15 & $285.415,94 * *$ \\
\hline ES 01 & 0,09 & $3.141,92$ & $3.075,74$ & $1,20^{*}$ & $1,12^{\mathrm{ns}}$ & 80,29 & 80,72 & $322.534,96 * *$ \\
\hline ES 23 & 0,04 & $2.470,59$ & $2.443,72$ & $1,03^{\mathrm{ns}}$ & $1,07^{\mathrm{ns}}$ & 93,86 & 94,03 & $-10.240,53^{\mathrm{ns}}$ \\
\hline VCP & 0,11 & $1.904,77$ & $1.755,75$ & $0,86^{\mathrm{ns}}$ & $0,74^{*}$ & 73,10 & 74,44 & $209.592,11 * *$ \\
\hline VSM & 0,11 & $2.421,43$ & $2.286,82$ & $1,17^{\mathrm{ns}}$ & $1,28 *$ & 87,21 & 87,97 & $134.233,12 *$ \\
\hline
\end{tabular}

${ }^{(1)} \hat{\beta}_{1}=\left(\mathrm{S}^{\mathrm{k}}+\right.$, variação em módulo entre os estimadores do coeficiente de inclinação estimados pelos métodos de mínimos quadrados e de regressão não paramétrica; $\hat{\beta}_{0 \mathrm{i}}^{*}$, intercepto (Eberhart \& Russel, 1966); $\hat{\beta}_{0 \mathrm{i}}$, intercepto (Theil, 1950); $\hat{\beta}_{\mathrm{li}}^{*}$, parâmetro de adaptabilidade pelo método de mínimos quadrados; $\hat{\beta}_{\mathrm{li}}$, parâmetro de adaptabilidade pela regressão não paramétrica; $\mathrm{R}_{\mathrm{t}_{\mathrm{i}}}^{2}$, coeficiente de determinação para o método proposto; $\mathrm{R}_{\mathrm{ER}}^{2}$, coeficiente de determinação pelo

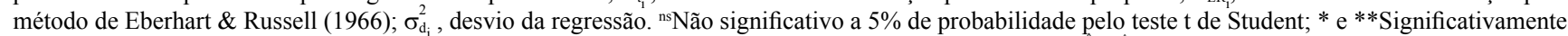

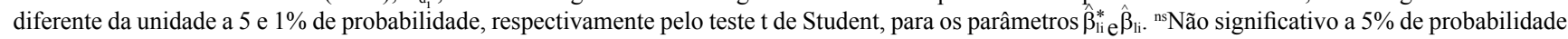
pelo teste $\mathrm{F} ; * \mathrm{e}^{* *}$ Significativo a 5 e $1 \%$ de probabilidade, respectivamente pelo teste $\mathrm{F}$, para o parâmetro $\sigma_{\mathrm{di}}^{2}$. 
método de regressão não paramétrica que no método de Eberhart \& Russell (1966) (Figuras 1 e 2).

A estimativa de estabilidade (Tabela 2) para os genótipos afetados pelos pontos extremos é feita por
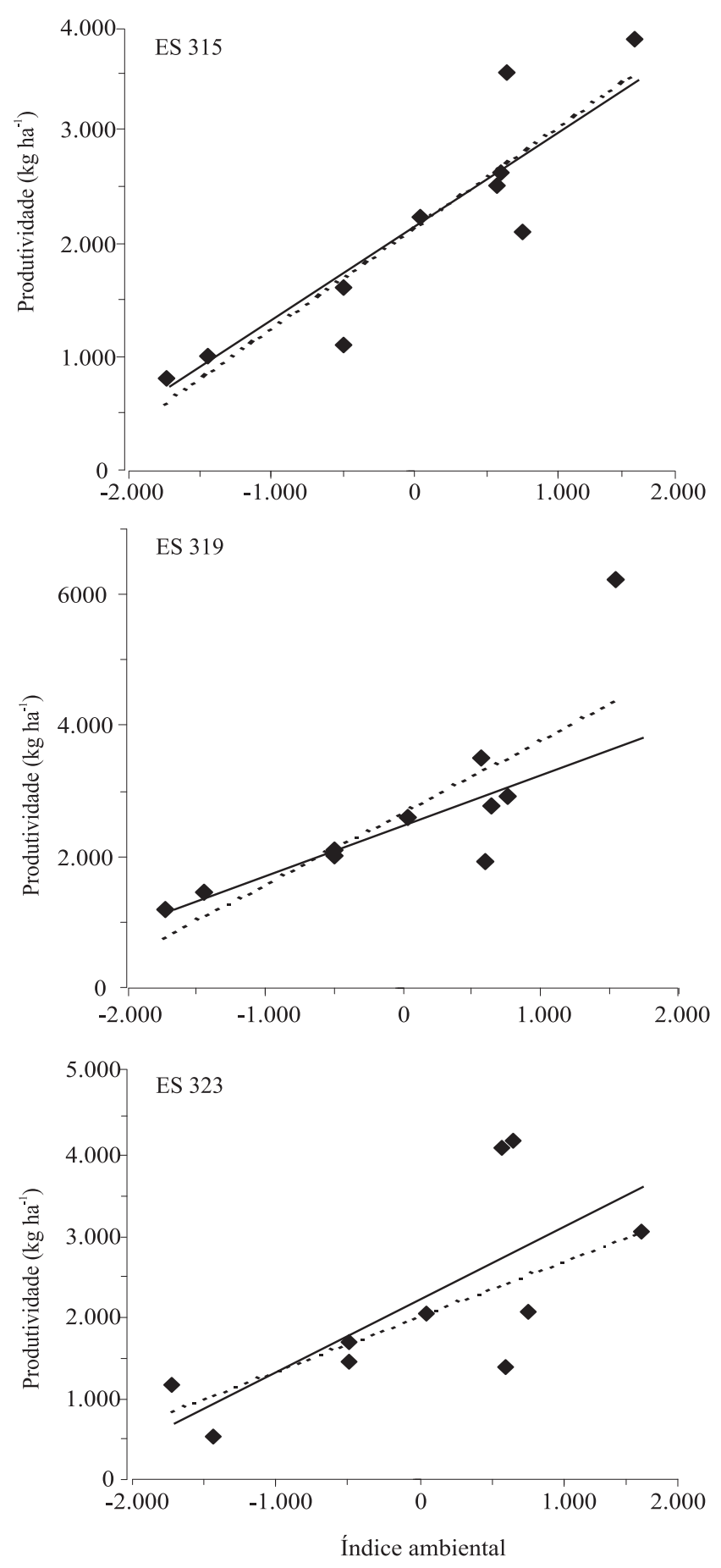

meio de $\mathrm{R}_{\mathrm{T}_{\mathrm{i}}}^{2}$, diferentemente do método proposto por Eberhart \& Russell (1966), que utiliza o desvio da regressão como estimativa. No método proposto, os genótipos ES 315, ES 36, ES 01, VCP e VSM foram
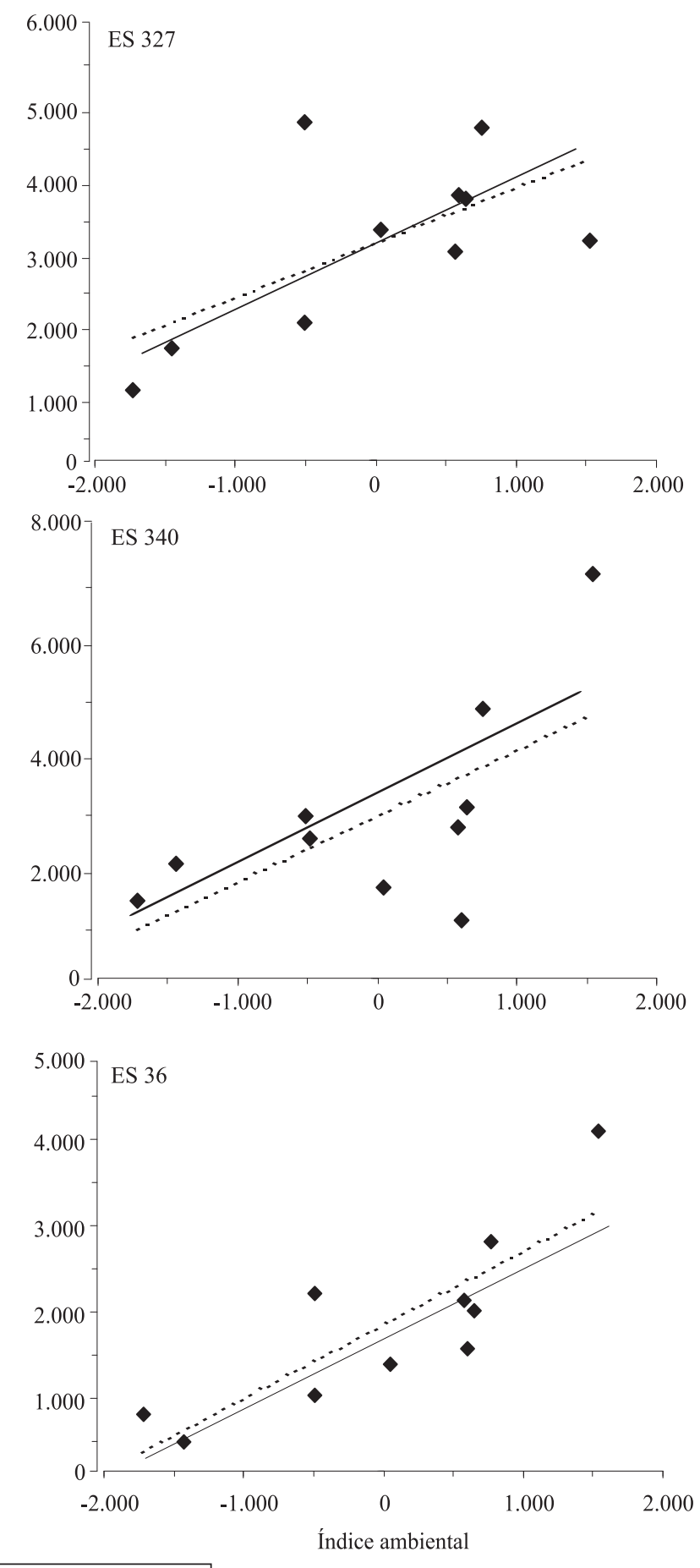

Figura 1. Retas estimadas pelos métodos de Eberhart \& Russell (1966) e Theil (1950), para os genótipos ES 315, ES 327, ES 319 , ES 340, ES 323 e ES 36. 
classificados como de estabilidade ou previsibilidade alta $\left(\mathrm{R}_{\mathrm{T}_{\mathrm{i}}}^{2}>70,00\right)$, enquanto os genótipos ES 319 , ES 323, ES 327 e ES 340 foram classificados como de estabilidade ou previsibilidade baixa. Com os resultados obtidos pela metodologia de Eberhart \& Russell (1966), todos esses genótipos analisados são considerados de estabilidade ou previsibilidade baixa.
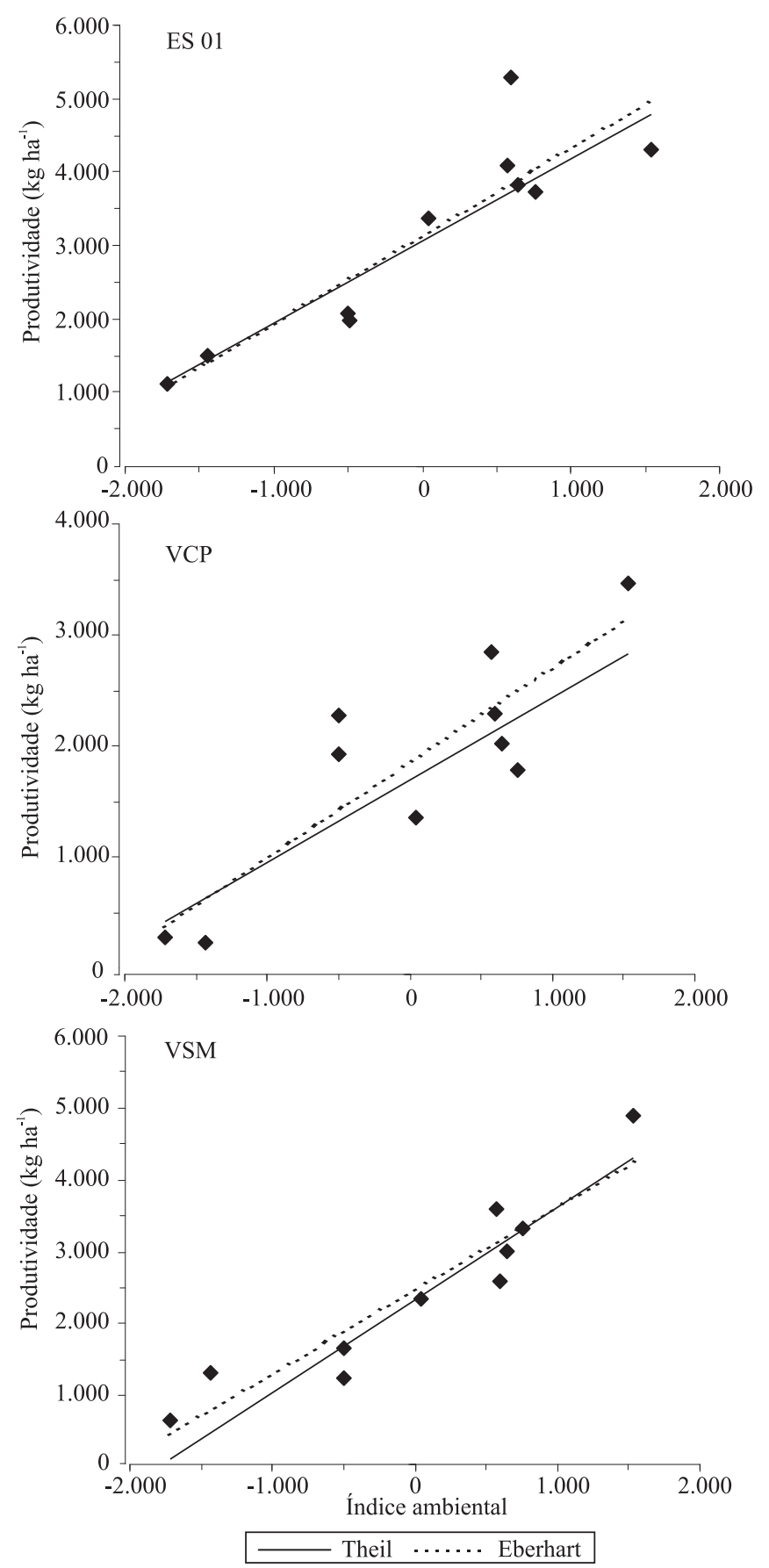

Figura 2. Retas estimadas pelos métodos de Eberhart \& Russell (1966) e Theil (1950), para os genótipos ES 01, VCP e VSM.
Sabe-se que nos métodos baseados em regressão linear simples o intercepto de cada genótipo (Tabela 2) é dado pela média dos genótipos em todos os ambientes, o que não acontece pelo método de regressão não paramétrica, no qual se estimou o intercepto a partir de medianas, tendo sido verificado que não existem grandes diferenças entre as estimativas pelos dois métodos.

Demonstrou-se que o uso do método de regressão não paramétrica é apropriado quando existem pontos extremos, em razão de o estimador de interesse via regressão não paramétrica ser menos sensível a pontos extremos que os estimadores clássicos obtidos pelo método dos mínimos quadrados ordinários (Sprent \& Smeeton, 2000). Isso ocorre porque os estimadores não paramétricos são calculados com uso de medianas, e o ponto extremo terá influência reduzida na estimação dos parâmetros, o que evita a má interpretação do parâmetro de adaptabilidade.

Do mesmo modo, a existência de pontos extremos causa problemas nas estimativas baseadas na medida não paramétrica de Lin \& Binns (1988), pois o parâmetro estimado por esse método é uma medida relativa a uma cultivar hipotética de adaptabilidade geral, cujo coeficiente de regressão é igual ou muito próximo à unidade (Cruz et al., 2003).

O resultado encontrado pela metodologia de Lin \& Binns (1988) não foi informativo, uma vez que alguns dos genótipos avaliados têm duplicidade de indicações (Tabela 3). É possível observar que o genótipo ES 327, afetado pelo ponto extremo, foi classificado como de adaptabilidade específica a ambientes desfavoráveis. Porém, é possível que o parâmetro tenha sido subestimado em razão da ação do ponto extremo nesse caso, pois, conforme o método não paramétrico proposto, ele foi de adaptabilidade geral. Outro exemplo é o genótipo ES 01, que classificou-se como de adaptabilidade específica a ambientes favoráveis quando analisado pela metodologia de Lin \& Binns (1988) e como de adaptabilidade geral pela metodologia não paramétrica. Fica evidente a redução da influência do ponto extremo na estimação do parâmetro de adaptabilidade e a maior facilidade de análise e interpretação pelo método de regressão não paramétrica em relação à metodologia de Lin \& Binns (1988). 
Tabela 3. Estimativas da estabilidade e adaptabilidade de 40 genótipos de café avaliados em dez ambientes, por meio da metodologia de Lin \& Binns (1988) ${ }^{(1)}$.

\begin{tabular}{lcrrr}
\hline Genótipo & Média & Pi geral & Pi favorável & Pi desfavorável \\
\hline ES 329 & $4.087,16$ & 323,62 & 372,21 & 250,74 \\
ES 328 & $3.592,72$ & 913,86 & 736,32 & $1.180,16$ \\
ES 336 & $3.591,51$ & 987,86 & $1.464,70$ & 272,58 \\
ES 337 & $3.507,93$ & $1.343,36$ & $1.297,83$ & $1.411,65$ \\
ES 339 & $3.183,27$ & $1.633,19$ & $1.965,10$ & $1.135,33$ \\
ES 327 & $3.235,27$ & $1.731,81$ & $2.508,89$ & 566,20 \\
ES 01 & $3.141,91$ & $1.794,96$ & $1.859,06$ & $1.698,79$ \\
ES 314 & $3.002,79$ & $2.000,19$ & $2.608,22$ & $1.088,14$ \\
ES 320 & $2.910,58$ & $2.155,48$ & $2.309,79$ & $1.924,03$ \\
ES 313 & $2.817,98$ & $2.159,49$ & $2.235,44$ & $2.045,56$ \\
ES 310 & $3.059,26$ & $2.228,58$ & $2.659,00$ & $1.582,94$ \\
ES 311 & $2.786,42$ & $2.303,69$ & $2.630,30$ & $1.813,78$ \\
ES 321 & $2.816,58$ & $2.367,45$ & $3.280,96$ & 997,19 \\
ES 325 & $2.744,88$ & $2.421,33$ & $2.692,84$ & $2.014,06$ \\
ES 307 & $2.889,74$ & $2.457,06$ & $2.464,88$ & $2.445,35$ \\
ES 335 & $2.684,65$ & $2.544,50$ & $3.262,25$ & $1.467,88$ \\
ES 316 & $2.736,44$ & $2.657,16$ & $3.502,27$ & $1.389,49$ \\
ES 340 & $2.985,45$ & $2.657,20$ & $3.837,12$ & 887,32 \\
ES 334 & $2.726,63$ & $2.664,66$ & $2.855,67$ & $2.378,15$ \\
ES 319 & $2.681,05$ & $2.769,32$ & $3.531,05$ & $1.626,72$ \\
ES 330 & $2.621,23$ & $2.781,16$ & $3.037,59$ & $2.396,53$ \\
ES 326 & $2.791,69$ & $2.785,88$ & $3.324,29$ & $1.978,26$ \\
ES 309 & $2.878,66$ & $2.810,59$ & $4.091,28$ & 889,57 \\
ES 332 & $2.623,07$ & $2.909,33$ & $3.427,04$ & $2.132,75$ \\
ES 308 & $2.795,90$ & $2.938,82$ & $4.081,38$ & $1.224,99$ \\
ES 23 & $2.470,59$ & $2.953,00$ & $3.441,97$ & $2.219,55$ \\
ES 331 & $2.628,48$ & $3.000,66$ & $4.273,09$ & $1.092,03$ \\
ES 312 & $2.534,10$ & $3.051,66$ & $4.421,37$ & 997,10 \\
VSM & $2.421,43$ & $3.169,20$ & $3.479,67$ & $2.703,50$ \\
ES 317 & $2.383,15$ & $3.291,35$ & $3.816,04$ & $2.504,33$ \\
ES 322 & $2.364,67$ & $3.323,66$ & $4.171,04$ & $2.052,59$ \\
ES 338 & $2.338,82$ & $3.444,06$ & $4.610,23$ & $1.694,80$ \\
ES 315 & $2.135,27$ & $3.978,19$ & $4.707,75$ & $2.883,85$ \\
ES 324 & $2.074,83$ & $4.044,45$ & $5.197,90$ & $2.314,26$ \\
ES 333 & $2.073,81$ & $4.139,45$ & $5.216,30$ & $2.524,17$ \\
ES 323 & $2.168,12$ & $4.403,01$ & $5.596,67$ & $2.612,52$ \\
ES 306 & $2.027,94$ & $4.452,39$ & $5.555,15$ & $2.798,26$ \\
VCP & $1.904,77$ & $4.595,25$ & $6.237,42$ & $2.132,00$ \\
ES 36 & $1.903,26$ & $4.642,95$ & $5.932,66$ & $2.708,38$ \\
ES 318 & $1.352,13$ & $6.852,66$ & $9.155,80$ & $3.397,96$ \\
\hline IVValors & Piv & &
\end{tabular}

${ }^{(1)}$ Valores de Pi divididos por 1.000

\section{Conclusões}

1. O método de regressão não paramétrica é menos influenciado por pontos extremos.

2. A utilização do método em que genótipos tenham resposta diferenciada em algum ambiente evita a má interpretação do parâmetro de adaptabilidade.

3. O método proposto não possui efeito de duplicidade de interpretação em comparação com a metodologia de Lin \& Binns (1988).

4. Os genótipos ES 311, ES 314, ES 317, ES 321, ES 322, ES 325, ES 330, ES 332, ES 333, ES 335, ES 336, ES 01 e ES 23 são classificados como de adaptabilidade geral e previsíveis de acordo com a metodologia proposta.

\section{Referências}

ALBRECHT, J.C.; VIEIRA, E.A.; SILVA, M.S. e; ANDRADE, J.M.V. de; SCHEEREN, P.L.; TRINDADE, M. da G.; SOARES SOBRINHO, J.; SOUSA, C.N.A. de; REIS, W.P.; RIBEIRO JÚNIOR, W.Q.; FRONZA, V.; CARGNIN, A.; YAMANAKA, C.H. Adaptabilidade e estabilidade de genótipos de trigo irrigado no Cerrado do Brasil Central. Pesquisa Agropecuária Brasileira, v.42, p.1727-1734, 2007.

CARNEIRO, P.C.S. Novas metodologias de análise da adaptabilidade e estabilidade de comportamento. 1998. $168 \mathrm{p}$. Tese (Doutorado) - Universidade Federal de Viçosa, Viçosa.

CRUZ, C.D. Programa Genes: biometria. Viçosa: UFV, 2006. 382p.

CRUZ, C.D.; CARNEIRO, P.C.S. Modelos biométricos aplicados ao melhoramento genético. Viçosa: UFV, 2003. v.2, 585p.

CRUZ, C.D.; REGAZZI, A.J.; CARNEIRO, P.C.S. Modelos biométricos aplicados ao melhoramento genético. 3.ed. Viçosa: UFV, 2004. v.1, 480p.

CRUZ, C.D.; TORRES, R.A. de A.; VENCOVSKY, R. An alternative approach to the stability analysis proposed by Silva and Barreto. Revista Brasileira de Genética, v.12, p.567-580, 1989.

EBERHART, S.A.; RUSSELL, W.A. Stability parameters for comparing varieties. Crop Science, v.6, p.36-40, 1966.

LIN, C.S.; BINNS, M.R. A superiority measure of cultivar performance for cultivar $\mathrm{x}$ location data. Canadian Journal of Plant Science, v.68, p.193-198, 1988.

MATSUO, E.; SEDIYAMA, T.; BARROS, H.B.; CRUZ, C.D.; ODA, M. do C.; TEIXEIRA, R. de C. Análise da estabilidade e previsibilidade da qualidade fisiológica de sementes de soja produzidas em Cristalina, Goiás. Acta Scientiarun. Agronomy, v.30, p.191-196, 2008.

MIRANDA, G.V.; SOUZA, L.V. de; GUIMARÃES, L.J.M.; NAMORATO, H.; OLIVEIRA, L.R.; SOARES, M.O. Multivariate analyses of genotype $\mathrm{x}$ environment interaction of popcorn. Pesquisa Agropecuária Brasileira, v.44, p.45-50, 2009.

NASCIMENTO, M.; CRUZ, C.D.; CAMPANA, A.C.M.; TOMAZ, R.S.; SALGADO, C.C; FERREIRA, R. de P. Alteração no método centroide de avaliação da adaptabilidade genotípica. Pesquisa Agropecuária Brasileira, v.44, p.263-269, 2009a.

NASCIMENTO, M.; FERREIRA, A.; CAMPANA, A.C.M.; SALGADO, C.C.; CRUZ, C.D. Multiple centroid methodology to analyze genotype adaptability. Crop Breeding and Applied Biotechnology, v.9, p.8-16, 2009 b.

PEREIRA, H.S.; MELO, L.C.; DEL PELOSO, M.J.; FARIA, L.C. de; COSTA, J.G.C. da; DÍAZ, J.L.C.; RAVA, C.A.; WENDLAND, A. Comparação de métodos de análise de adaptabilidade e estabilidade fenotípica em feijoeiro-comum. Pesquisa Agropecuária Brasileira, v.44, p.374-383, 2009.

PORTO, W.S.; CARVALHO, C.G.P. de; PINTO, R.J.B. Adaptabilidade e estabilidade como critérios para seleção de genótipos de girassol. Pesquisa Agropecuária Brasileira, v.42, p.491-499, 2007. 
SPRENT, P.; SMEETON, N.C. Applied nonparametric statistical methods. $4^{\text {th }}$ ed. Boca Raton: Chapman and Hall, 2000. 480p.

THEIL, H. A rank-invariant method of linear and polynomial regression analysis. Indagationes Mathematicae, v.12, p.85-91, 1950.
VASCONCELOS, E.S. de; BARIONI JÚNIOR, W.; CRUZ, C.D.; FERREIRA, R. de P.; RASSINI, J.B.; VILELA, D. Seleção de genótipos de alfafa pela adaptabilidade e estabilidade da produção de matéria seca. Acta Scientiarum. Agronomy, v.30, p.339-343, 2008.

Recebido em 30 de outubro de 2009 e aprovado em 30 de dezembro de 2009 